

\title{
Remarks on the issue of time and complex numbers in canonical quantum gravity
}

\author{
J.L. ROSALES * \\ Fakultät für Physik, \\ Universität Freiburg,Hermann-Herder-Strasse 3 \\ D-79104 Freiburg, Germany.
}

(January 9, 2018)

\begin{abstract}
Upon using some special example in the homogeneous cosmological model we develop the idea that, as a result of the arbitrariness of the factor ordering in Wheeler-DeWitt equation, gauge phases can not, in general, being completely removed from the wave functional in quantum gravity. The latter may be conveniently described by means of a remnant complex term in WDW equation depending on the factor ordering. Taking this equation for granted we can obtain WKB complex solutions and, therefore, we should be able to derive a semiclassical time parameter for the Schrödinger equation corresponding to matter fields in a given classical curved space. 4.60.+n,03.65Bz,04.20.Fy
\end{abstract}

Typeset using REVTEX

*E-mail: rosales@phyq1.physik.uni-freiburg.de 


\section{Introduction.}

The precise formulation of quantum theory of gravity is not yet known. However, such a theory should be developed in order to address some fundamental questions in Physics like what happens at the Planck era of the universe or during the final evolution of a black hole.

As approximate first step, we can cast general relativity into Hamiltonian form and then, formally quantize it according to the canonical quantization rules. The intention is to derive a wave equation analogously to the Schrödinger picture of quantum mechanics; we thus obtain a set of independent wave equations where the three-geometries and matter fields play the role of configuration space. The main result of this approach is the canonical hamiltonian constraint or Wheeler-DeWitt equation (WDW) [1]

$$
H \Psi\left(g^{3} ; \phi\right)=0
$$

As a general rule, apart from a gauge fixing which would involve a global arbitrary phase factor, the hamiltonian $H$ is supposed to be a real operator in configuration space. An additional observation is that time is absent and so the state of the universe (the object to which we apply WDW equation) is stationary.

Yet, taking canonical quantum gravity for granted, we should understand the connection of this framework to more standard concepts, particularly we must be able to derive the approximation of Quantum Field Theory in curved Spacetime. The recovery of this limit can be obtained by using an expansion, for the complex solutions of (1) in powers of the Planck mass (assuming that this represents a mass far beyond the relevant energy scale for the matter fields). This is just a Born-Oppenheimer approximation (see e.g. [2]). In recent years [3], [G], [5], however, it has been notice that there exist no sufficient reason for the election of complex waves as the solutions of such a real equation and, in general, we could speculate about special prescriptions for the wave function [6]. In this brief report, on the other hand, we will try to show that the real feature of (1) might be just a consequence of some fine tuning selection corresponding to the operator factor ordering ambiguity of the theory and that, in some more general cases, arbitrary phases can not be completely 
removed from the quantum theory. The latter would result in a remnant complex term within the definition of the quantum Hamiltonian operator; from this new tentative complex formulation of quantum gravity, a semiclassical time would be implemented automatically after the direct Born-Oppenheimer expansion.

On the other hand, it has been claimed that the mechanism of decoherence would account for an effective branching of a given real solution into its complex components [7] [8], [9], which could perhaps be interpreted as a symmetry breaking analogously to the case of chirality in sugar molecules [10]. This would make possible, for an observer in a semiclassical universe, to detect no interference whatsoever between the terms that appear in such a superposition of waves. Finally, some coarse grained density matrix for the relevant variables (e.g. the scale factor of the cosmological model) is found to be almost diagonal after cosmological evolution (upon tracing out gravitational fluctuations and matter fields which are not measured by such a -classical- observer). This is possible since gravity always couples to matter (which is now responsible for self-measurement of the universe). Thus, in spite of the fact that Wheeler-DeWitt equation is invariant under complex conjugation, the actual states should be intrinsically complex and the whole process behaves as a phase transition in the cosmological evolution. On the other hand, this argument by no means avoids the possible presence of real superpositions in the quantum state (which has not been measured since it represents the global information of the state of the universe, including gravitational fluctuations and matter fields). Only from this state we are allowed to derive the approximate Schrödinger equation. Taking this into account, we must, perhaps, consider some more direct possibilities in order to justify the emergence of some semiclassical time parameter from the asymptotic expansion about these very special complex WKB states.

Quantum gravity in the homogeneous model.

Our general discussion will be done for the minisuperspace approximation in the homogeneous cosmological model. Therefore, as far as we are concerned on the general features corresponding to this model, let us collect the general properties of its well known classical and quantum theories [11, this model has the advantage of working on a one dimensional 
space of variables for the set of Hamilton field equations and seems to be a good approximation for the universe we observe.

In the homogeneous model of the universe we can also take into account the coupling features of an scalar massive field $\phi$. It is defined by means of the metric given by

$$
d s^{2}=-N(t)^{2} d t^{2}+a(t)^{2} d \Omega_{3}^{2}
$$

$N$ is the lapse function, $a$ is the scale factor and $d \Omega_{3}^{2}$ is the standard three-sphere metric. Expressing the scalar field as $\left(2 \pi^{2}\right)^{1 / 2} \phi$ with the quadratic potential $2 \pi^{2} m^{2} \phi^{2}$ the action is $\left(H_{0}^{2}=\frac{\Lambda}{3}, \Lambda\right.$ being the cosmological constant)

$$
S=-\frac{1}{2 \sigma^{2}} \int d t N a^{3}\left\{\frac{\dot{a}^{2}}{N^{2} a^{2}}-\frac{1}{a^{2}}+H_{0}^{2}-\sigma^{2}\left[\frac{\dot{\phi}^{2}}{N^{2}}-m^{2} \phi^{2}\right]\right\}
$$

Here, $\sigma$ denotes the Planck length in geometrodynamical units. Varying the action with respect to $N$ lead to the constraint

$$
H=\frac{N}{2 a^{3}}\left\{-a^{2} \sigma^{2} p_{a}^{2}+p_{\phi}^{2}+\frac{1}{\sigma^{2}}\left[H_{0}^{2} a^{6}-a^{4}\right]+m^{2} \phi^{2} a^{6}\right\}=0
$$

where $p_{a}=-\frac{\dot{a}}{\sigma^{2} N} a, p_{\phi}=\frac{a^{3}}{N} \dot{\phi}$.

In the conformal gauge the hamiltonian is given by

$$
H=\frac{1}{2}\left\{-\sigma^{2} p_{a}^{2}+2 u(a)\right\}+H_{m}\left(\phi, p_{\phi}\right)
$$

where the matter Hamiltonian is given by

$$
H_{m}\left(\phi, p_{\phi}\right)=\frac{1}{2}\left\{\frac{p_{\phi}^{2}}{a^{2}}+a^{4} m^{2} \phi^{2}\right\}
$$

and $u(a)=\frac{1}{2 \sigma^{2}}\left(H_{0}^{2} a^{4}-a^{2}\right)$.

It leads to the Wheeler-DeWitt equation for the state functional of the universe written as

$$
\left\{\sigma^{2} a^{2-p} \frac{\partial}{\partial a} a^{p} \frac{\partial}{\partial a}-\frac{\partial^{2}}{\partial \phi^{2}}+V^{2}(a ; \phi)\right\} \Psi(a ; \phi)=0
$$

where $V^{2}(a ; \phi)=2 a^{2} u(a)+a^{6} m^{2} \phi^{2}$, is the general relativity potential for the homogeneous model. The parameter $p$ takes into account some of the factor ordering ambiguity of the 
theory. For the semiclassical derivation of the Schrödinger equation for the matter fields the parameter $p$ is of little importance, however, following the suggestion of Dirac [12], we have to keep it in mind in order to define the correct quantum theory, moreover, as we will see, the possibilities of the quantum theory are not completely exhausted from the previous formulation. In order to clarify this, let us proceed to discuss Dirac's observation in the context of the homogeneous cosmological model.

We should start from assuming that the quantum theory does not need to be a direct result of the classical formulation we dispose and, therefore, we must take special care in developing its physical possibilities.

In order to examplify our discussion we could search for some special mini-superspace Hamiltonian obtained from Legendre-transforming some Lagrangian coming from the addition in (3) of some special gauge phase. The latter, of course, will always preserve classical equations of motion but, in general, would also involve additional products of quantum (non-conmuting) operators in such a new Hamiltonian. A relevant example of the previous idea follows from selecting the Hamiltonian given by

$$
H^{c}=-\frac{\sigma^{2}}{2} p_{a}^{2}-\frac{1}{2 \sigma^{2}} a^{2}-k p_{a} H_{0} a^{2}+H_{m}\left(\phi, p_{\phi}\right)
$$

where $k$ is some real parameter and the product of non-conmuting operators is shown explicitly. We have required the presence of some cosmological constant, $H_{0}$, from dimensional considerations. The linear term in the momentum would lead to the expected complexification of the constraint when expressing it quantum mechanically.

It is immediately obvious that, for $k= \pm 1$, the difference between the Legendre transformed Lagrangians corresponding to $H$ and $H^{c}\left(L(H)=\left(p_{a} \partial / \partial p_{a}-1\right) H\right)$ is just a total derivative

$$
L(H)=L\left(H^{c}\right)+\frac{d}{d t} \alpha(a)
$$

where $\alpha(a)=-k H_{0} a^{3} / 3 \sigma^{2}$. This make spurious the choice of the dynamics. In this case (upon taking $k=1$ ), we get the constraint 


$$
\left\{\frac{\sigma^{2}}{2} a^{-p} \frac{\partial}{\partial a} a^{p} \frac{\partial}{\partial a}-i H_{0} a^{2-q} \frac{\partial}{\partial a} a^{q}-\frac{a^{2}}{2 \sigma^{2}}+H_{m}\left(\phi, p_{\phi}\right)\right\} \tilde{\Psi}=0
$$

Since the physically meaningful quantity is $|\Psi|^{2}$ (or $|\tilde{\Psi}|^{2}$ ) we should develop the gauge transformation a little bit further upon removing the resulting phase

$$
\tilde{\Psi}=\Psi_{c} e^{-i \alpha(a)}
$$

The wave function $\Psi_{c}$ now satisfies, from (9)

$$
\left\{\sigma^{2} a^{2-p} \frac{\partial}{\partial a} a^{p} \frac{\partial}{\partial a}-\frac{\partial^{2}}{\partial \phi^{2}}+V_{c}^{2}(a ; \phi)\right\} \Psi_{c}(a ; \phi)=0
$$

where

$$
V_{c}^{2}(a ; \phi)=V^{2}(a ; \phi)+i(p+2-q) H_{0} a^{3}
$$

and a complex term depending on the factor ordering is shown explicitly; of course, it has not classical interpretation and comes directly from the quantum theory.

We should take into account that (11) has only complex solutions. We are allowed, therefore, to obtain directly WKB-like solutions and, thus, the approximate Schrödinger equation for the matter field. In order to achieve this let us write the wave function as follows

$$
\Psi_{c}(a ; \phi)=e^{i S(a)} \psi(a ; \phi)
$$

where $S(a)=\frac{S_{0}}{\sigma^{2}}+S_{1}+\sigma^{2} S_{2}+\ldots$; then, inserting (13) in (11) we obtain, for any $p$ and $q$, up to $O\left(\sigma^{0}\right)$, the Hamilton-Jacobi equation for a matter free universe.

$$
\left(\frac{\partial S_{0}}{\partial a}\right)^{2}+a^{2}-H_{0}^{2} a^{4} \approx 0
$$

Therefore, as a result of the WKB regime of the solution the wave is strongly correlated about particular classical configurations.

Also, $O\left(\sigma^{2}\right)$ equations lead to

$$
i \frac{\partial \psi(a ; \phi)}{\partial \tau} \approx H_{m} \psi(a ; \phi)
$$


where we have defined an intrinsic time derivative

$$
\frac{\partial S_{0}}{\partial a} \frac{\partial}{\partial a} \equiv \frac{\partial}{\partial \tau}
$$

Here, $\tau$ is nothing more than an affine parameter which labels the points along the trajectories about which the wave function is peaked. In order to define such an intrinsic semiclassical time we have not required decoherence of macroscopic states in a real superposition since those wave functions no longer satisfy equation (11).

Moreover, the only place where the complex factor is recovered is in $S_{1}$. We obtain for it,

$$
2 \frac{\partial S_{1}}{\partial a} \frac{\partial S_{0}}{\partial a}=i \frac{\partial^{2} S_{0}}{\partial a^{2}}+i \frac{p}{a} \frac{\partial S_{0}}{\partial a}+i \gamma a
$$

where, $\gamma=(p+2-q) H_{0}$ is a new constant.

In order to understand the meaning of $\gamma$, we must, perhaps, relate its very origin from the conditions of normalizability of the wave function in minisuperspace. To see this, let us assign a probability meaning to $\Psi$ in the sense of Hawking and Page [13], i.e., $|\Psi|^{2}$ is proportional to the probability of finding a 3-surface $S$ with metric $h_{i j}$ and matter field configuration $\phi$; Moreover, they suggested that $p=1$ solves a part of the factor ordering problem, since, in the previous case, WDW equation becomes hyperbolic in configuration space. On the other hand, the behaviour of the semiclassical wave functional at $a \ll H_{0}^{-1}$ is given, for any $\gamma$ by

$$
|\Psi|^{2} \sim a^{-2} e^{-a^{2} / \sigma^{2}}
$$

therefore, the norm should be defined in terms of the following finite quantity

$$
<\Psi \mid \Psi>=\int d a d \phi a^{2} \Psi^{*}(a ; \phi) \Psi(a ; \phi)<\infty
$$

and this is just possible for the semiclassical wave functions only in case that $\gamma \neq 0$. This may be seen from the behaviour of $|\Psi|^{2}$ at infinity derived from (17) and (14),i.e.,

$$
|\Psi|^{2} \sim a^{-3-\frac{\gamma}{H_{0}}}
$$


which does not depend on the value of the cosmological constant. Now, from (19), if $\gamma$ would vanish exactly, the norm $\langle\Psi \mid \Psi\rangle$ diverges as the logarithm of the scale factor and, therefore, we are not allowed to assign a probability amplitude meaning to $\Psi$. The norm of the wave functional converges only if $\gamma>0$. Thus, we might undestand the very meaning of the new constant $\gamma$ from the requirements of the quantum theory. On the other hand, $\gamma$ may reach a value very close to zero but it should not be strictly zero.

Finally let us make some additional remarks indicating that there exits a quantum spacetime governed by $\Psi_{c}$ and that the problem of obtaining its classical properties still persists; this would lead to the mechanism of self-measurement of the universe as established in [14] and [8], thus, only an effective classical universe is observed since the different many branches described by the functional state $\Psi_{c}$ decohere. This is required since, even if one restrict attention to a single WKB component of a general complex superposition, one can not speak of a classical spacetime because WKB wave functionals are spread out over all configuration space 周.

\section{Summary and conclusions}

One of the Prima Facie questions in quantum gravity concerns with the meaning of its classical limit, i.e., how to construct the corresponding quantum analogue of the classical theory of gravity we dispose [15]. In a practical sense the matter is usually decided on an $a d$ hominum basis. Here we have written down two specific proposals for the quantum gravity theory of the universe i.e., it could be described either by a real functional $\Psi$ as well as by, perhaps, its complex generalization given by $\Psi_{c}$. We motivated the second possibility in order to recover some semiclassical intrinsic time parameter in the Schrödinger equation for the matter field. The state $\Psi_{c}$ might also contain new physics since the complex potential has not classical interpretation. Nevertheless, we have been more concerned on the appropriated specification of the semiclassical limit in this quantum theory and, as far as this requires a

\footnotetext{
${ }^{*}$ I thank to Claus Kiefer for this comment
} 
superselection rule which eliminates real superposition states, we have stressed the selection of the complex state described by the solution of the complex wave equation. Since this was found just from a very special minisuperpace, a general analysis of the behaviour of this imaginary term in superspace is still necessary.

Acknowledgments

The author wishes to express his thanks to David Hochberg, José Luis Sánchez-Gómez and Claus Kiefer for discussing the issue raised in this paper. I also wish to express my warmest gratitude to Professor Hartmann Römer in extending to me the hospitality of the University of Freiburg, where part of this work was done. This work is supported by the Spanish Ministry of Sciences scholarship number EX95 08960718. 


\section{REFERENCES}

[1] B.S. DeWitt, Phys. Rev., 160, 1113, (1967)

[2] T. Banks, Nucl. Phys., B249, 332 (1985)

[3] C. Kiefer, Phys. Rev., D47, 5414, (1993)

[4] J.B. Barbour, Phys. Rev., D47, 5422, (1993)

[5] J.B. Barbour and L. Smolin, Can Quantum Mechanics be sensibly applied to the Universe as a whole?, Yale report, 1988 (unpublished).

[6] A discussion of the very nature the solutions would lead us to consider the specification of some special boundary condition for the wave functional; see, for instance J.B. Hartle and S.W. Hawking, Phys. Rev. D28, 2960 (1983) and A. Vilenkin, Phys. Rev., D33, 3560 (1986).

[7] C. Kiefer, Class. Quantum. Grav., 9, 147,(1992)

[8] J. Halliwell, Phys. Rev., D39, 2912,(1989)

[9] C. Kiefer, Phys. Rev., D46, 1658 (1992)

[10] H.D. Zeh, "Decoherence and measurements". In Stochastic evolution of quantum states in open systems and measurement processes, edited by L.Diósi and B. Lukács, Singapore:World Scientific.

[11] S.W. Hawking, Nucl. Phys., B239, 257 (1984)

[12] P.A.M. Dirac, Lectures on Quantum Field Theory, Academic Press Inc., pages 41 to 43, (1966)

[13] S.W. Hawking and D.N. Page, Nucl. Phys., B264, 185 (1986)

[14] C. Kiefer, Class. Quantum Grav., 4, 1369 (1987) 
[15] C.J. Isham, Prima Facie Questions in Quantum Gravity, Imperial College preprint TP/93-94/1 (1993) 